\title{
Contralateral Negative Bold Responses in the Motor Network during Subthreshold High-Frequency Interleaved TMS-fMRI over the Human Primary Motor Cortex
}

Tsuyoshi Shimomura ${ }^{1 *}$, Minoru Fujiki ${ }^{1}$, Hiroshi Ohba ${ }^{1}$, Takanori Kochiyma ${ }^{2}$, Kenji Sugita' ${ }^{1}$ Hiroyuki Matsuta ${ }^{1}$, Yukari Kawasaki ${ }^{1}, K^{2}$ Kohei Oonishi ${ }^{1}$, Hirotaka Fudaba ${ }^{1}$ and Thoru Kamida ${ }^{1}$

${ }^{1}$ Deparment of Neurosurgery, Oita University Faculty of Medicine, Oita, Japan

${ }^{2}$ Brain Activity Imaging Center, Advanced Telecommunications Research Institute International, Soraku-gun, Japan

\begin{abstract}
Objective: Transcranial Magnetic Stimulation (TMS) is a noninvasive technique that stimulates a localized brain region underneath a coil. Recently, the application of repetitive Transcranial Magnetic Stimulation (rTMS) to the primary motor cortex (M1) has been used to promote functional recovery in stroke patients with hemiparesis. Highfrequency rTMS ( $5 \mathrm{~Hz}$ or greater) was applied over the affected hemisphere to reactivate hypoactive regions. Because of recent advances in MRI technology and, in particular, the gradient coils, high-frequency whole brain rTMS-fMRI is possible. The use of interleaved rTMS-fMRI during stimulation at a frequency of $5 \mathrm{~Hz}$ aids in understanding how the brain is modulated during stimulation.
\end{abstract}

Method: We measured BOLD signal changes in whole brain during interleaved rTMS-fMRI (5Hz) using 3T MRI. Image processing and statistical analyses were carried out using the Statistical Parametric Mapping (SPM8) software.

Results: Distinct BOLD signal changes extending to the remote motor network during $5 \mathrm{~Hz}-\mathrm{rTMS}$ over the M1 were successfully demonstrated using interleaved rTMS-fMRI. Negative BOLD responses were observed in the contralateral M1, the dorsal premotor cortex (PMd), and the bilateral supplementary motor cortex (SMA), even though no significant BOLD signal changes were observed in the stimulated M1. The negative BOLD responses gradually became marked during $(3$ mins $57 \mathrm{sec}$ ). Therefore, long-lasting plastic changes may occur within 4 mins. Negative BOLD responses in remote regions away from the directly stimulated M1 suggest that subthreshold high frequency rTMS over the motor cortex induced neuromodulation via neuronal networks.

Conclusion: The remarkable negative BOLD responses in the contralateral M1 induced by high-frequency rTMS may be useful for identifying a treatment strategy involving M1 stimulation.

Keywords: High frequency rTMS; Negative BOLD; fMRI; Motor cortex; SPM; Neuromodulation; Motor network

\section{Introduction}

Transcranial Magnetic Stimulation (TMS) can stimulate brain underneath a coil noninvasively [1]. Because of the unique natures, TMS has been widely used to basic neuroscience research and clinical applications. Nevertheless, it is no fully understood that how to TMS influence to both excitatory and inhibitory neuronal circuits. Recently, the application of rTMS of the primary motor cortex (M1) has been reported to promote functional recovery in stroke patients with hemiparesis due to induced neuroplasticity [2]. Functional recovery was obtained when high-frequency rTMS ( $5 \mathrm{~Hz}$ or greater) was applied over the affected hemisphere to activate hypoactive regions [3-5].

rTMS has been considered to modulate the sensorimotor network by either direct M1 stimulation or by an indirect effect through transsynaptic connections. However, the precise mechanism of its facilitative and inhibitory effects on the sensorimotor network remains uncertain [6]. Functional Magnetic Resonance Imaging (fMRI) studies by M1 TMS demonstrated widespread increases and decreases in Blood Oxygen Level-Dependent (BOLD) signals in cortical and subcortical areas [6-9]. These studies have showed the cortico-cortical and corticosubcortical connections that regulate the sensorimotor network with multisynaptic modulation.

The aim of this study was to establish high frequency (greater than $5 \mathrm{~Hz}$ ) whole brain interleaved TMS-fMRI by taking advantage of recent advances in MRI equipment and, in particular, the gradient coil system.
We also investigated BOLD signal responses during $5 \mathrm{~Hz}$-rTMS. In addition, we describe the effects of subthreshold high-frequency rTMS (which has recently been used in clinics to promote functional recovery in stroke patients) in directly stimulated M1 and areas distantly connected by neuronal networks. rTMS-fMRI during stimulation at a frequency of $5 \mathrm{~Hz}$ is important for understanding treatment strategies using TMS. In this study, we selected $5 \mathrm{~Hz}$-rTMS for study because 5 $\mathrm{Hz}$-rTMS is widely used as a high frequency rTMS in clinical studies and is used as the basic frequency in theta burst stimulation.

\section{Materials and Methods}

Six healthy 26 to 36 year-old (mean 33.3; SD 4.3 years) male volunteers, judged according to the Edinburgh Handedness Inventory to be right-handed, participated in this study. None of the subjects

*Corresponding author: Dr. Tsuyoshi Shimomura, Department of Neurosurgery Oita University Faculty of Medicine, Oita, 879-5593 Japan, Tel: +81-97-586-5490; Fax+81-97-586-6324; E-mail: simomura@oita-u.ac.jp

Received December 06, 2018; Accepted December 26, 2018; Published December 31, 2018

Citation: Shimomura T, Fujiki M, Ohba H, Kochiyma T, Sugita K, et al. (2018) Contralateral Negative Bold Responses in the Motor Network during Subthreshold High-Frequency Interleaved TMS-fMRI over the Human Primary Motor Cortex. J Neurol Neurophysiol 9: 478. doi:10.4172/2155-9562.1000478

Copyright: (c) 2018 Shimomura T, et al. This is an open-access article distributed under the terms of the Creative Commons Attribution License, which permits unrestricted use, distribution, and reproduction in any medium, provided the original author and source are credited. 
Citation: Shimomura T, Fujiki M, Ohba H, Kochiyma T, Sugita K, et al. (2018) Contralateral Negative Bold Responses in the Motor Network during Subthreshold High-Frequency Interleaved TMS-fMRI over the Human Primary Motor Cortex. J Neurol Neurophysiol 9: 478. doi:10.4172/21559562.1000478

had a history of neurological or psychiatric illness, and none was on psychotropic medications. The study protocol was approved by the Ethics Committee of the Oita University Faculty of Medicine. The subjects were fully informed about the experimental procedures, and all subjects participated in the experiment after providing written informed consent.

\section{TMS and electromyography (EMS)}

TMS and EMS settings were achieved according to the previous reports [9]. The motor hot spot was identified where obtained a maximal motor response in the right First Dorsal Interosseous (FDI) muscle by TMS. A MRI-compatible figure-eight-coil with a $70 \mathrm{~mm}$ outer-wing diameter (MR coil, Magstim, Whitland, Wales, UK) was positioned tangential to the scalp of the subjects at the motor hot spot in a 3-Tesla whole-body MRI scanner equipped with a Circular Polarization (CP) head coil (Siemens Magnetom Verio, Erlangen, Germany). The coil was connected to a biphasic stimulator (Rapid²Plus, Magstim, Whitland, Wales, UK) via a $10 \mathrm{~m}$ cable. Motor-Evoked Potentials (MEPs) were recorded from the right FDI with silver/silver chloride surface electrodes with a ground electrode on the dorsal surface of the right wrist, using a BrainAmp system (Brain Products GmbH, Germany) [9]. The TMS coil was fixed to the scanner bed with a custom-made coil holder, and wrapped in bubble wrap. Foam pads were used to minimize head motion during scanning. The resting motor threshold (RMT) was defined for each individual as the percentage of stimulator output that elicited MEPs were $>50 \mu \mathrm{V}$ peak-to-peak amplitude in the FDI at rest in more than 5 of 10 successive trials [10]. The active motor threshold, which is the threshold of detection under approximately $5 \%$ maximal voluntary muscle contraction, was also defined (data not shown).

\section{Image acquisition}

A time course series of 96 volumes was acquired with a $\mathrm{T} 2{ }^{*}$ weighted, single-shot, gradient-Echo Planar Imaging (EPI) sequence. Each volume comprised 24 slices, with a $4 \mathrm{~mm}$ slice thickness and a $1 \mathrm{~mm}$ gap, and encompassed almost the entire cerebral and superior cerebellar cortices. Images were acquired in the axial plane. The Repetition Time (TR) was 2,400 ms, the Echo Time (TE) was $23 \mathrm{~ms}$, and the flip angle was $90^{\circ}$. The Field of View (FOV) was $210 \mathrm{~mm}$, the voxel size was $3 \times 3 \times 4 \mathrm{~mm}$, the slice gap was $1 \mathrm{~mm}$, and the matrix size was $70 \times 70 \mathrm{~mm}$. The total acquisition times were 3 mins $57 \mathrm{sec}$, including periods for signal equilibration. T1-weighted structural images were acquired with a 3-D magnetization prepared rapid gradient echo (MPRAGE) in the sagittal plane. The TR was $1,900 \mathrm{~ms}$, the TE was 2.53 $\mathrm{ms}$, the Inversion Time (TI) was $900 \mathrm{~ms}$, and the flip angle was $9^{\circ}$. The FOV was $210 \mathrm{~mm}$, the slice thickness was $1 \mathrm{~mm}$, the slice gap was 0.5 $\mathrm{mm}$, the number of slices collected was 176 , and a $192 \times 192 \times 256$ matrix facilitated localization.

Interleaved TMS-fMRI was accomplished as previously described [3-5]. In a previous report, the minimum waiting period between a TMS pulse and a subsequent EPI acquisition was $69 \mathrm{~ms}$ [7]. Each image was acquired for $91 \mathrm{~ms}$ in that report. In recent years, MRI technologies have advanced; thus, the TE and duration required for each image acquisition have decreased. Therefore, in this study, functional images were acquired every $100 \mathrm{~ms}$, and TMS pulses were applied immediately after each image acquisition. The stimulation timing was controlled by Presentation software (Neurobehavioral systems, Albany, CA) on a personal computer that was synchronized with the MRI scanner via transistor-transistor logic pulses converted from the default optical signals of the scanner. In a phantom study, the best timing of the TMS pulse was determined based on the TMS recording and imaging artifacts using the BrainAmp system. The chosen stimulation frequencies of 5 $\mathrm{Hz}$ (TMS every two image acquisitions) required $60 \mathrm{~ms}$ waiting periods between TMS pulses and subsequent image acquisitions; this design allowed for unperturbed MRI acquisition, as confirmed by phantom experiments. The basic experimental protocol followed a block design. A rest-TMS-rest cycle was repeated eight times and each epoch was $9.6 \mathrm{sec}$. Therefore, the TMS period was $9.6 \mathrm{sec}$, and the interval of each TMS period was $19.2 \mathrm{sec}$ (Figure 1). "Rest" indicates that the subject was resting quietly with his eyes closed, and "TMS" indicates TMS at $90 \%$ of RMT.

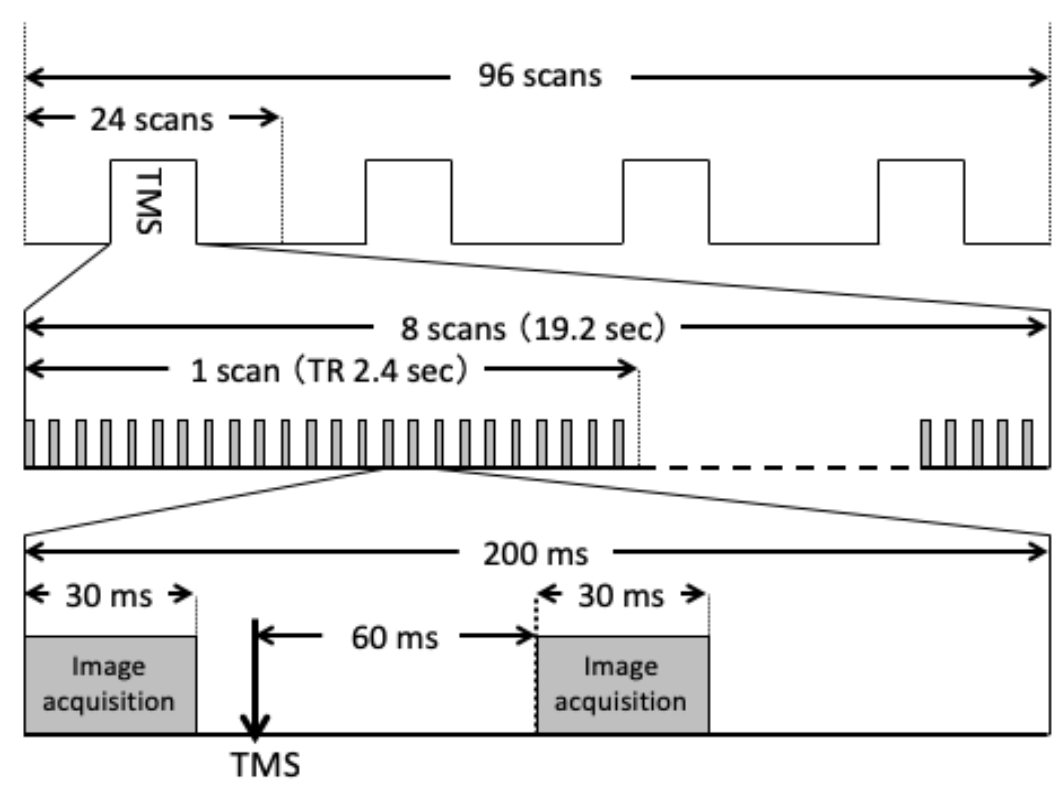

Figure 1: Ninety-six scans were obtained over a period of $3 \mathrm{~min} 57 \mathrm{sec}$, consisting of four epochs of TMS. TMS was applied at $5 \mathrm{~Hz}$ (every two image acquisitions) for $19.2 \mathrm{sec}$. Image acquisition was achieved within $30 \mathrm{~ms}$, and TMS was applied $60 \mathrm{~ms}$ before the RF pulse used for the next image acquisition. 
Citation: Shimomura T, Fujiki M, Ohba H, Kochiyma T, Sugita K, et al. (2018) Contralateral Negative Bold Responses in the Motor Network during Subthreshold High-Frequency Interleaved TMS-fMRI over the Human Primary Motor Cortex. J Neurol Neurophysiol 9: 478. doi:10.4172/21559562.1000478

\section{Image processing}

Image processing and statistical analyses were performed using the Statistical Parametric Mapping (SPM8) software (Wellcome Department of Cognitive Neurology, London, UK). All EPI images were spatially realigned, normalized and smoothed using the Montreal Neurological Institute (MNI) EPI template and an 8-mm full-width, half-maximum Gaussian filter. At the individual subject level, each task was modeled as a boxcar function, which was subsequently convolved with the canonical hemodynamic response function $[8,11]$. Data were further treated using a high-pass filter with a cutoff of $128 \mathrm{~s}$ and Grand mean scaling. T-contrast was calculated by differences in activation between each task and the resting blocks. The subsequent whole-brain, multisubject analysis was performed by a fixed model analysis. The height threshold for the $\operatorname{SPM}(\mathrm{T})$ was set at $\mathrm{p}<0.05$ and was corrected for multiple comparisons across the entire brain. The cytoarchitectonic nomenclature of significant brain activity was determined according to the SPM anatomy toolbox [12]. To detect the time-varying modulation of task-related BOLD responses, we used a first-order time modulation model in SPM8. F-contrast was used to test over all the effects of interest (i.e., the task-related changes and their linear increase or decrease over time). The height threshold for the $\operatorname{SPM}\{\mathrm{F}\}$ was set at $\mathrm{p}<0.05$ and was corrected for multiple comparisons across the whole brain with inclusion masked by the main effect ( $T$-contrast, $\mathrm{p}<0.05$, uncorrected).

The percent signal changes in hemodynamic signals were estimated in each subject using Automatic Anatomical Labeling (AAL) ROIs with the Marseille region of interest toolbox (http://marsber.souceforge. net/). The percent signal changes of the bilateral precentral cortex (M1), the bilateral supplementary motor cortex (SMA), and the bilateral thalamus (THA) were sampled. The data were divided into first and second half sessions. ROI analyses were carried out in $5 \mathrm{~Hz}-\mathrm{rTMS}-\mathrm{A}$ and $5 \mathrm{~Hz}$-rTMS-B groups.

\section{Results}

The phantom study demonstrated the presence of artifacts caused by TMS at the time of image acquisition (Figure 2A). TMS was applied after image acquisition and $60 \mathrm{~ms}$ before the RF pulse used for the next image acquisition; no artifact was observed in any image (Figure 2B). When asked immediately after the experiment, none of the subjects reported any side effects from the experimental procedure, with the exception of a mild, painless sensation from the contact of the TMS coil with their head. The EPI images of the subjects' brains contained no visible dropouts. We performed a quality check on each sequence and found no artifacts in any images acquired using the interleaved TMSfMRI. No muscle twitch in the right FDI muscle was observed during rTMS-fMRI via video monitoring or with EMG monitoring using the BrainAmp system with MRI-compatible filters.

During high frequency rTMS, distinct positive and negative BOLD responses were induced and spread to the distant motor network areas, despite the lack of significant BOLD signal changes in the directly stimulated left M1. Distinct significantly increased BOLD responses were found in the bilateral S2 and auditory regions. In addition, a slight activation was observed in the bilateral thalamus, the bilateral putamen, and the cerebellum. Remarkable extended, decreased BOLD responses were observed in the right M1 (contralateral to the stimulated side), the right dorsal premotor cortex (PMd), the bilateral SMA, the bilateral prefrontal cortices, and the bilateral parieto-occipital cortices (Figure 3, Table 1).

Time-varying modulations of the BOLD responses to the $5 \mathrm{~Hz}$ rTMS were observed in the right M1, the left SMA, and the bilateral parieto-occipital cortices using time modulation analysis with the inclusive mask of the negative BOLD responses (Figure 4). By plotting the fitted response, it was observed that this negative BOLD response decreased gradually. Time-varying modulations were not found in the positive BOLD responses to the $5 \mathrm{~Hz}-\mathrm{rTMS}$.

The percent signal change in the bilateral M1 observed during the second-half rTMS was significantly lower than that observed during the first-half rTMS (Figure 5). The percent signal change in the bilateral SMA observed during the second-half rTMS was lower than that observed during the first-half rTMS, but this effect was not significant.
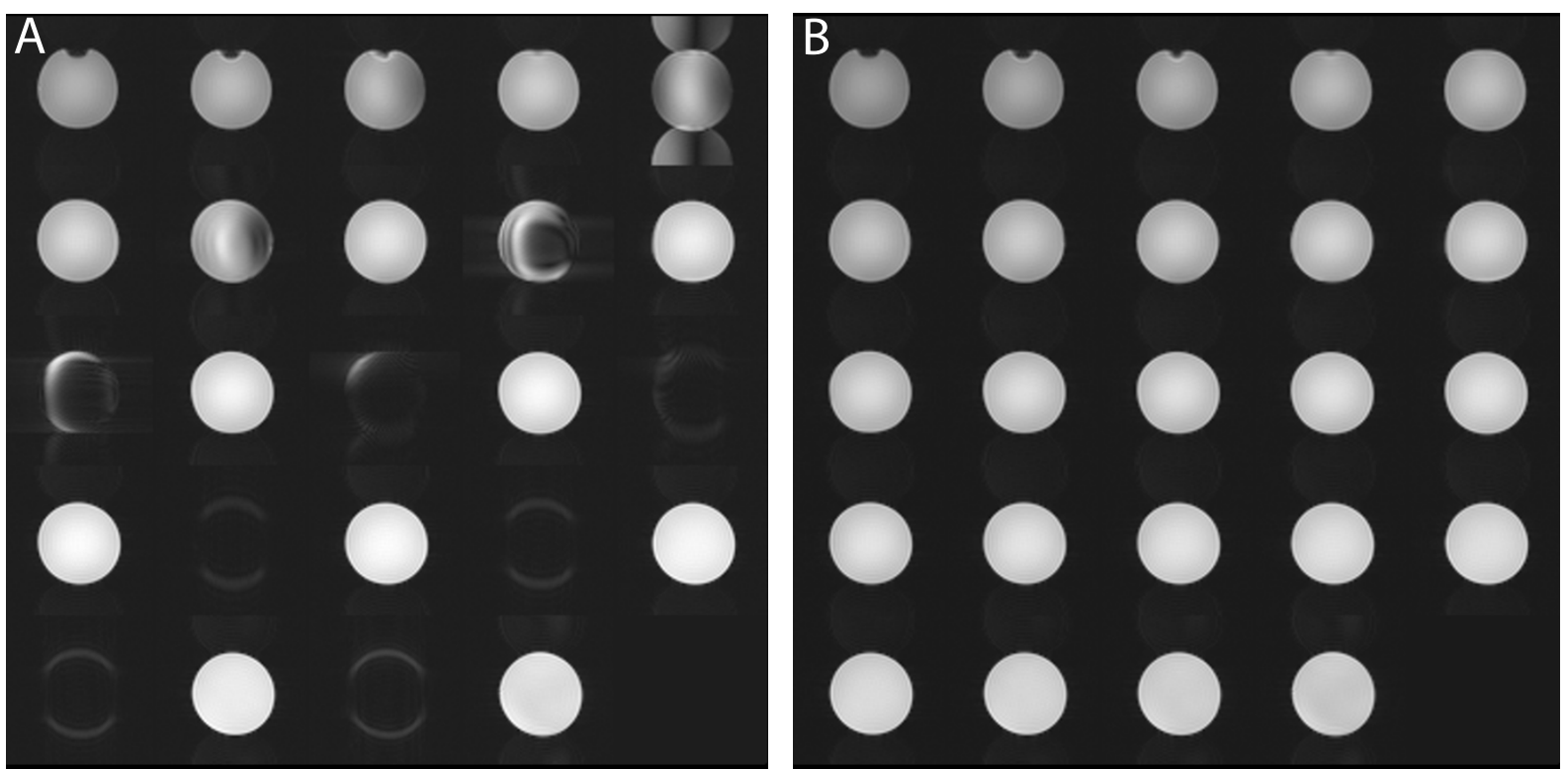

Figure 2: (A) Visible dropouts caused by TMS were observed in almost every two images when TMS was applied during image acquisition; (B) No artifacts caused by TMS were observed when TMS was applied $60 \mathrm{~ms}$ before the RF pulse used for the next image acquisition. 
Citation: Shimomura T, Fujiki M, Ohba H, Kochiyma T, Sugita K, et al. (2018) Contralateral Negative Bold Responses in the Motor Network during Subthreshold High-Frequency Interleaved TMS-fMRI over the Human Primary Motor Cortex. J Neurol Neurophysiol 9: 478. doi:10.4172/21559562.1000478

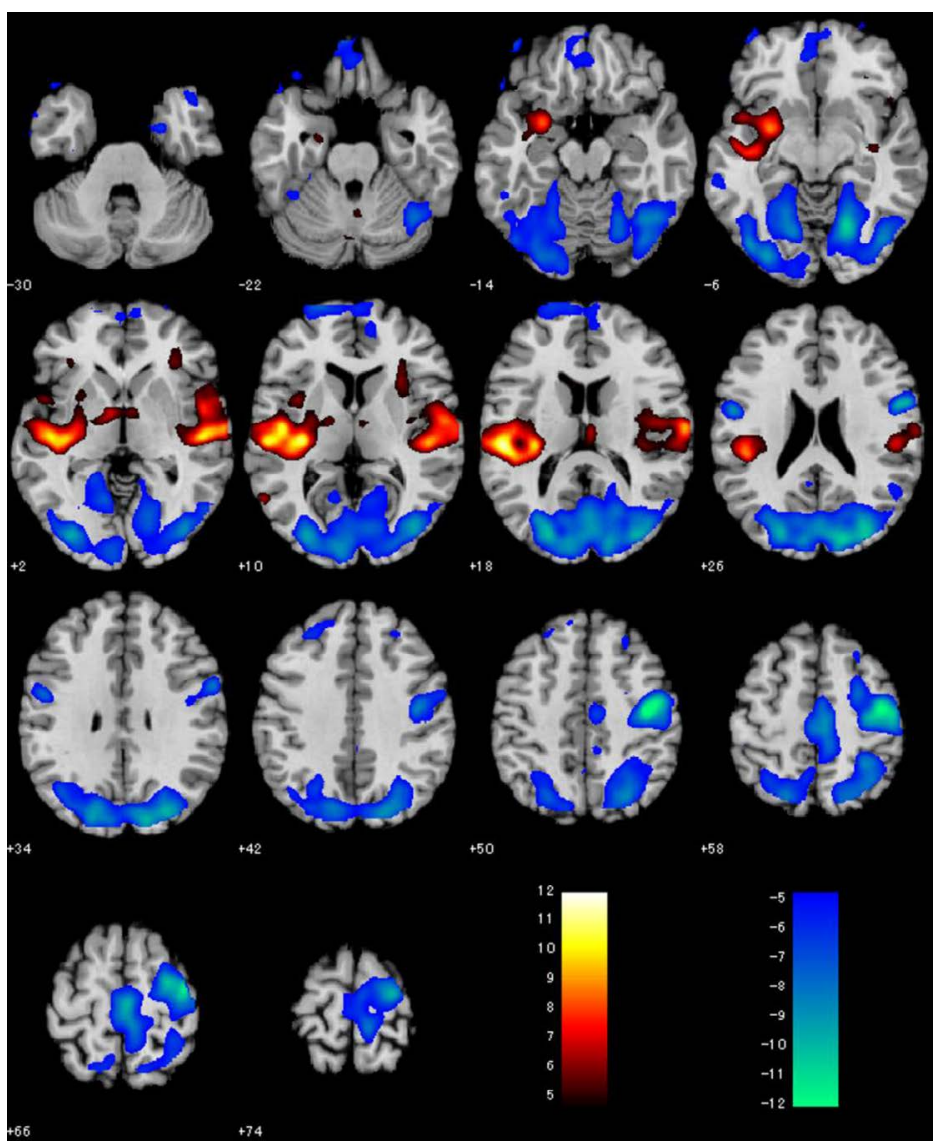

Figure 3: Activation obtained from a group analysis (six subjects, $p<0.05$, FWE) of responses to subthreshold rTMS at $90 \%$ of the Resting Motor Threshold (RMT) onto axial sections of a standard reference brain (Montreal Neurological Institute, MNI). The left hemisphere is shown on the left. Activation, i.e., positive BOLD MRI responses or signal increases, are coded in red-yellow; BOLD signal decreases are coded in blue. Activation obtained from a group analysis of responses to 5 - $\mathrm{Hz}$ subthreshold rTMS (Height threshold T=4.747157, p<0.05, FEW; Extent threshold k=0).
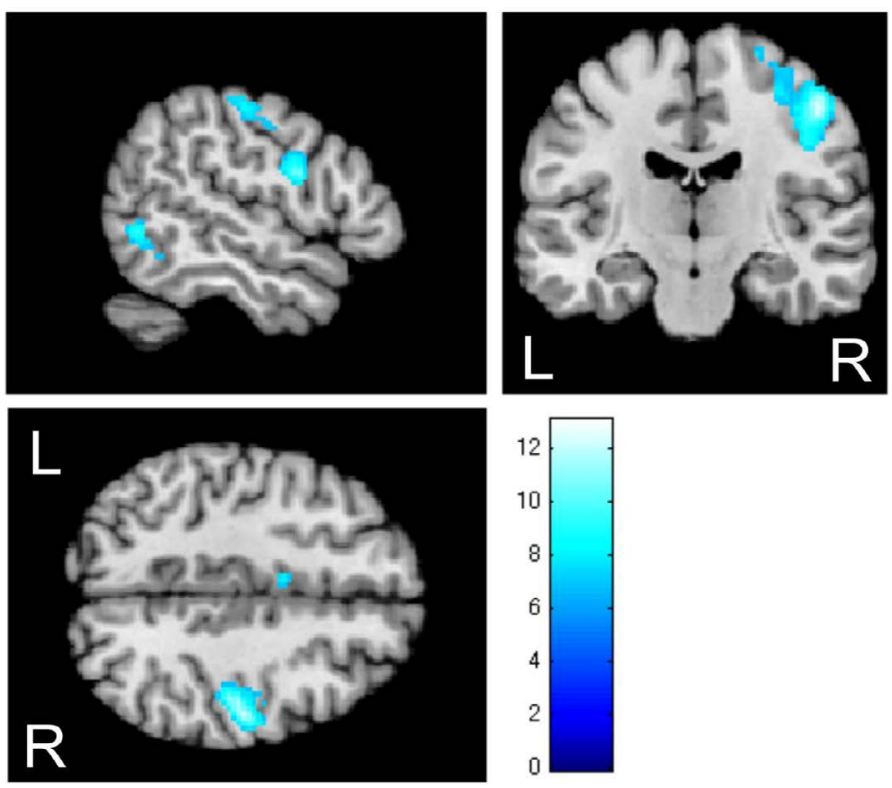

Figure 4: Activation obtained from a group analysis of responses to $5-\mathrm{Hz}$ subthreshold $\mathrm{rTMS}$ using time modulation with an inclusive mask of the negative main effect (Height threshold $\mathrm{F}=6.645297, \mathrm{p}<0.05$, FEW; Extent threshold $\mathrm{k}=0$ ). 
Citation: Shimomura T, Fujiki M, Ohba H, Kochiyma T, Sugita K, et al. (2018) Contralateral Negative Bold Responses in the Motor Network during Subthreshold High-Frequency Interleaved TMS-fMRI over the Human Primary Motor Cortex. J Neurol Neurophysiol 9: 478. doi:10.4172/21559562.1000478

Page 5 of 8

\begin{tabular}{|c|c|c|c|c|c|c|c|}
\hline \multirow{2}{*}{\multicolumn{3}{|c|}{$\begin{array}{c}\text { Active clusters } \\
\text { (functional anatomy) }\end{array}$}} & \multirow[t]{2}{*}{ Volume $\left(\mathrm{mm}^{3}\right)$} & \multicolumn{3}{|c|}{ MNI Coordinate } & \multirow[t]{2}{*}{ T-value } \\
\hline & & & & $\mathbf{x}$ & $y$ & $\mathbf{z}$ & \\
\hline \multicolumn{8}{|c|}{ Positive BOLD } \\
\hline 1 & L & Superior marginal gyrus (OP1/OP4) & 37784 & -54 & -26 & 16 & 12.27 \\
\hline 2 & $\mathrm{R}$ & Superior marginal gyrus (OP1/OP4) & 22648 & 50 & -26 & 6 & 10.67 \\
\hline 3 & L & middle temporal gyrus & 576 & -58 & -64 & 8 & 6.88 \\
\hline 4 & $\mathrm{R}$ & putamen & 2144 & 30 & 14 & 8 & 6.21 \\
\hline 5 & L & insula & 344 & -34 & 26 & 4 & 5.71 \\
\hline 6 & $\mathrm{R}$ & cerebellum (lobule VI) & 112 & 2 & -60 & -22 & 5.39 \\
\hline 7 & L & cerebellum (lobule VI) & 136 & -2 & -78 & -24 & 5.22 \\
\hline \multicolumn{8}{|c|}{ Negative BOLD } \\
\hline 1 & $\mathrm{R}$ & precentral gyrus (Area 4a) & 206592 & 42 & -18 & 52 & -13.31 \\
\hline 2 & L & precentral gyrus ( Area $4 p$ ) & 2352 & -52 & -6 & 28 & -8.68 \\
\hline 3 & $\mathrm{~L}$ & superior frontal gyrus & 12680 & -22 & 64 & 12 & -7.75 \\
\hline 4 & $\mathrm{~L}$ & temporal lobe & 808 & -46 & 24 & -38 & -7.66 \\
\hline 5 & $\mathrm{~L}$ & middle frontal gyrus & 712 & -44 & 54 & -12 & -7.07 \\
\hline 6 & $\mathrm{R}$ & inferior frontal gyrus & 1520 & 50 & 4 & -38 & -6.75 \\
\hline 7 & $\mathrm{R}$ & hippocampal gyrus & 464 & 24 & -4 & -32 & -6.47 \\
\hline 8 & L & middle frontal gyrus & 1408 & -26 & 34 & 42 & -6.18 \\
\hline 9 & L & temporal pole & 448 & -50 & 22 & -20 & -6.14 \\
\hline 10 & L & middle temporal gyrus & 792 & -54 & -46 & -10 & -5.79 \\
\hline 11 & $\mathrm{R}$ & middle frontal gyrus & 736 & 26 & 20 & 56 & 5.54 \\
\hline 12 & L & middle temporal gyrus & 120 & -56 & 2 & -28 & -5.53 \\
\hline 13 & L & cerebellum (lobule VI) & 168 & -28 & -38 & -26 & -5.29 \\
\hline 14 & $\mathrm{R}$ & superior orbital gyrus & 240 & 28 & 62 & -4 & -5.25 \\
\hline 15 & $R$ & fusiform gyrus & 120 & 34 & -34 & -28 & -5.1 \\
\hline
\end{tabular}

Table 1: Results from the group-level statistical parametric mapping analysis: BOLD responses during 5-Hz-rTMS

\section{Discussion}

Recent advances in MRI technology, especially gradient coil systems, has made possible to perform 5-Hz rTMS-fMRI. Images were acquired every $100 \mathrm{msec}$, and the whole brain was scanned in $2.4 \mathrm{sec}$. We chose to use $5 \mathrm{~Hz}$-rTMS because $5 \mathrm{~Hz}$ is the standard frequency for clinical TMS therapy and is the basic frequency of theta burst stimulation. This rTMS-fMRI protocol will therefore lead to theta-burst rTMS-fMRI.

The present results demonstrated positive BOLD responses in the bilateral S2, the bilateral auditory regions, the bilateral thalamus, and the cerebellum as the result of $5-\mathrm{Hz}$ interleaved subthreshold rTMSfMRI. Similar to the results found in previous reports [3,4], activation of the directly stimulated M1 was not observed. Clear negative BOLD responses were detected in the contralateral M1, the PMd, and the bilateral SMA while using the rTMS-fMRI frequency protocols. The finding of negative BOLD responses in regions that are remote from the directly stimulated M1 but functionally connected to the motor network suggests that neuronal modulations were induced by the subthreshold high-frequency rTMS across the motor cortex. This effect occurred despite the absence of significant BOLD signal responses in the directly stimulated left M1.

It is generally accepted that positive BOLD signal changes demonstrate increased brain activity [13]. In addition to positive BOLD signal changes, many studies have shown negative BOLD responses in areas such as the occipital cortex during various visual tasks [14,15], motor tasks [16,17], electrical stimulation of the median nerve [18], and TMS [6,7]. Previous investigations have provided indirect evidence that regions exhibiting negative BOLD responses are associated with neuronal suppression $[14,15,17,19]$. Furthermore, a non-human primate study involving simultaneous fMRI and electrophysiological recordings in monkeys reported direct associations between neural suppression and negative BOLD responses [20]. Our imaging data demonstrated that no significant activation of the ipsilateral M1 occurred while using high-frequency subthreshold rTMS. The absence of significant BOLD signal changes in the stimulated M1 has been noted previously and is suggested to be primarily due to the lack of afferent feedback from actual muscle movements induced by the TMS pulses [6,7]. Based on previously recommended safety guidelines [21], suprathreshold TMS was not used in this study.

Electrophysiological data have shown that subthreshold stimulation activates cortical circuitry, even if it fails to elicit the cortico-spinal output necessary for a muscle twitch [22,23]. Conversely, paired pulse experiments in which a small initial stimulus is used to condition the response to a larger test stimulus have clearly shown that subthreshold TMS can affect the excitability of cortical circuits $[7,23]$. Although Intracortical Inhibition (ICI) is partly regulated by GABA via postsynaptic GABAA-receptor activation [24], Intracortical Facilitation (ICF) is likely a more complex phenomenon because more than one neurotransmitter (i.e., glutamate, dopamine, and GABA) may be involved [25]. Thus, subthreshold pulses induce the trans-synaptic excitation of cortical neurons via interneurons. It is known that an increased distance results in insufficient sensitivity for the detection of subtle BOLD MRI signal changes at the stimulation site. Bestmann et al. speculated that this phenomenon is likely due to the self-canceling of inhibitory and excitatory processes, leading to negligible net synaptic activity with no hemodynamic output, together with the occurrence of altered hemodynamic response characteristics that may not be detected using common response functions [7]. These authors advocated a model in which local hemodynamic changes that are evoked at the site 
Citation: Shimomura T, Fujiki M, Ohba H, Kochiyma T, Sugita K, et al. (2018) Contralateral Negative Bold Responses in the Motor Network during Subthreshold High-Frequency Interleaved TMS-fMRI over the Human Primary Motor Cortex. J Neurol Neurophysiol 9: 478. doi:10.4172/21559562.1000478

Page 6 of 8
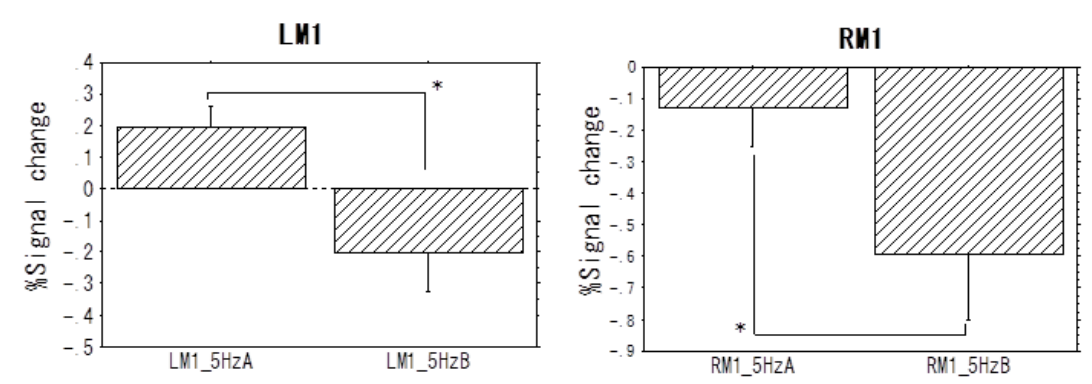

LSMA
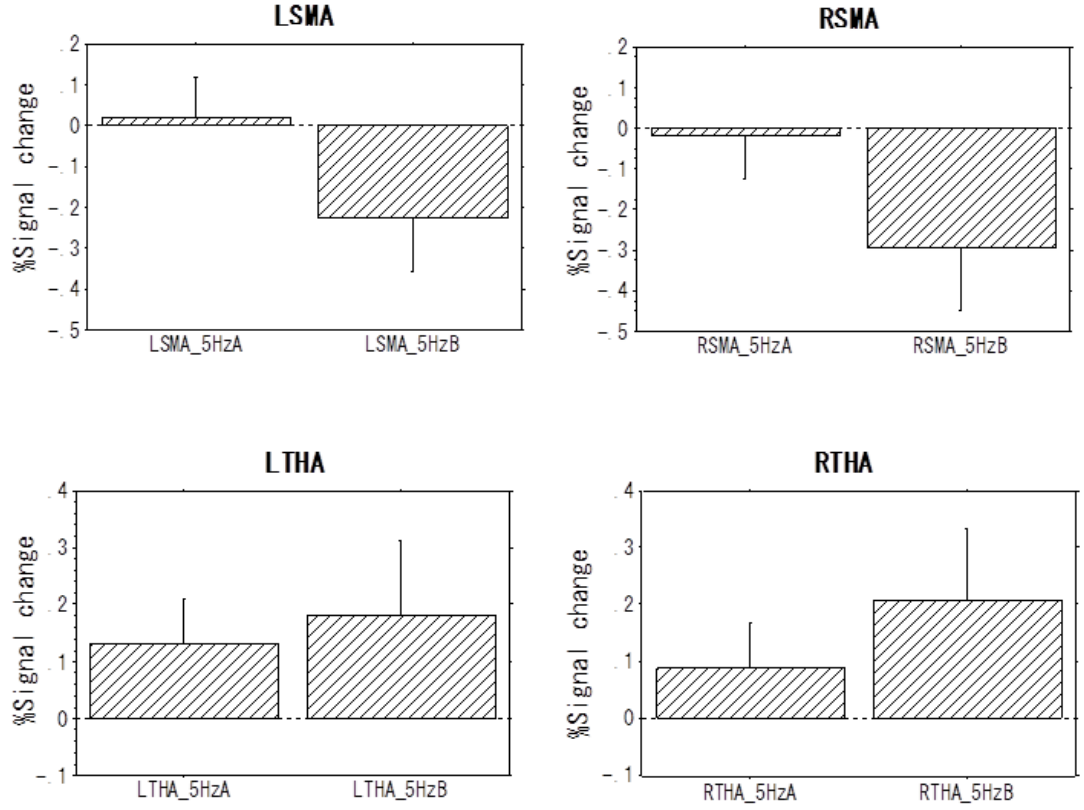

Figure 5: Stimulus-response profiles in the directly stimulated left M1 and remote areas during high-frequency rTMS. ${ }^{*} p<0.05$ (estimated using Student's t test). The error bars represent the standard error of the mean. M1: primary motor cortex; SMA: supplementary motor cortex; THA: thalamus; L: left; R: right.

of stimulation by subthreshold rTMS do not exceed the background physiological "noise" level. In our study, the percent signal change of the left M1 during $5 \mathrm{~Hz}$-rTMS-A revealed a slight increase in activity, followed by a significant decrease that became negative during $5 \mathrm{~Hz}$ rTMS-B. This activity resulted in a lack of any overall signal change during $5 \mathrm{~Hz}-\mathrm{rTMS}$. Furthermore, a recent study demonstrated that $5 \mathrm{~Hz}-\mathrm{rTMS}$ produces an increase in the Cortical Silent Period (CSP) duration that is not affected by repetitive paired pulse TMS [26]. CSP reflects the activation of postsynaptic GABAB-receptors, presumably on intracortical interneurons [27]. The coexistence of inhibitory and excitatory neuronal intracortical modulation at the directly stimulated site may have led to the observed fluctuating BOLD signals and may have resulted in the absence of significant activity increases in the left M1.

Similar to the findings of previous reports [6,7], we observed markedly negative BOLD responses in the contralateral M1 during high-frequency rTMS. Interhemispheric Inhibition (IHI) is a wellknown phenomenon that was first described by Ferbert et al. [28]. In this phenomenon, a conditioning TMS to M1 reduces the EMG activity produced by a second pulse to the contralateral M1 within an Interstimulus Interval (ISI) of 6-50 ms [29]. This event is likely to occur at the cortical level $[28,29]$ and is mediated through excitatory commissural neurons that have a propagation time of approximately 10-15 ms, exciting local inhibitory interneurons in the contralateral M1 [30]. Subsequent study reported that IHI between homologous M1s at ISI of $10 \mathrm{~ms}$ (short latency IHI; SIHI) and of $40 \mathrm{~ms}$ (long latency IHI; LIHI) may have different physiological origins [31]. SIHI/LIHI is widely distributed among motor related cortical areas, and suggests that this inhibitory drive may contribute to unimanual and coordinated bimanual movements at different stages of motor control, including the decision making, movement selection and execution [32]. Palmer et al reported that the firing of layer 5 pyramidal neurons in rat somatosensory cortex due to contralateral sensory stimulation was inhibited for hundreds of milliseconds when paired with ipsilateral stimulation. The inhibition acted directly on apical dendrites via layer 1 interneurons but was silent in the absence of pyramidal cell firing, relying on metabotropic inhibition of active dendritic currents recruited during neuronal activity [33]. IHI has also been observed during transcranial electrical cortex stimulation (TES) [29]. When a contralateral conditioning stimulus preceded the test stimulus by $10 \mathrm{~ms}$, the subsequent ipsilateral BOLD signal was significantly reduced. The negative BOLD responses in the contralateral M1 observed during high-frequency subthreshold rTMS were likely caused by IHI, which was mediated via excitatory commissural neurons acting on local inhibitory interneurons in the 
Citation: Shimomura T, Fujiki M, Ohba H, Kochiyma T, Sugita K, et al. (2018) Contralateral Negative Bold Responses in the Motor Network during Subthreshold High-Frequency Interleaved TMS-fMRI over the Human Primary Motor Cortex. J Neurol Neurophysiol 9: 478. doi:10.4172/21559562.1000478

ipsilateral M1. A near-infrared spectroscopy study demonstrated that TMS using continuous theta bursts over M1 elicited a significant oxy$\mathrm{Hb}$ decrease in the contralateral M1 [34].

Recently, the application of rTMS of the primary motor cortex has been reported to promote functional recovery in stroke patients with hemiparesis owing to induced neuroplasticity [35]. Functional recovery was obtained both when low-frequency rTMS (around $1 \mathrm{~Hz}$ ) was applied over the disinhibited, unaffected hemisphere to restore defective inhibition [36] and when high-frequency rTMS (5 Hz or more) was applied over the affected hemisphere to activate hypoactive regions $[37,38]$.

Our imaging data also revealed distinctly negative BOLD responses of the bilateral SMA. Conversely, positive as well as slightly negative BOLD responses of the SMA, without emphasis, have been previously reported [6,7]. Negative BOLD responses of the SMA were clear, and these effects are likely to have been associated with the negative BOLD responses observed in the contralateral M1. The SMA is densely interconnected with the M1 through the motor loop, one of the basal ganglia-thalamocortical connections [39]. The negative BOLD responses of the bilateral $\mathrm{M} 1$ may lead to the negative BOLD responses of the bilateral SMA through the multisynaptic neuronal network of the motor loop. Our findings demonstrated that gradually decreased BOLD responses to the $5 \mathrm{~Hz}$-rTMS were observed in the right $\mathrm{M} 1$ and the left SMA using time modulation analysis with the inclusive mask of the negative BOLD responses. Further, the time course of the percent signal change in each session demonstrated a gradually decreased BOLD response in the bilateral M1. Long-lasting plastic changes can occur within 4 mins during $5 \mathrm{~Hz}$-rTMS-fMRI.

In this study, we observed markedly decreased BOLD responses in the contralateral M1 during high frequency rTMS, despite a lack of significant BOLD signal changes in the stimulated M1. We speculate that neuronal modulation of the decreased activity in the contralateral M1 might have decreased the TCI from the contralateral M1 to the ipsilateral M1, which might in turn have increased the excitability of the ipsilateral M1. However, further studies are needed to test this hypothesis. The robust negative BOLD response in the contralateral M1 that was induced by high-frequency rTMS could provide promising alternative treatment strategies using M1 stimulation.

In conclusion, we successfully demonstrated distinct BOLD signal changes that extended to the remote motor network during $5 \mathrm{~Hz}-\mathrm{rTMS}$ over the M1 using interleaved rTMS-fMRI. The remarkable negative BOLD responses in the contralateral M1 induced by high-frequency rTMS might have diminished TCI from the contralateral to the stimulated M1.

\section{Acknowledgements}

Declaration of interest: The authors declare that no conflicts of interest exist that could be perceived as prejudicing the impartiality of the research reported Funding: This work was supported by a grant from the Japan Science and Technology Agency and the Ministry of Education, Culture, Sports, Science, and Technology.

\section{References}

1. Huang YZ, Edwards MJ, Rounis E, Bhatia KP, Rothwell JC (2005) Theta burst stimulation of the human motor cortex. Neuron 45: 201-206.

2. Lefaucheur JP (2006) Stroke recovery can be enhanced by using repetitive transcranial magnetic stimulation (rTMS). Neurophysiol Clin 36: 105-115.

3. Chang WH, Kim YH, Bang OY, Kim ST, Park YH, et al. (2010) Long-term effects of rTMS on motor recovery in patients after subacute stroke. J Rehabil Med 42: $758-764$
4. Kim YH, You SH, Ko MH, Park JW, Lee KH, et al. (2006) Repetitive transcrania magnetic stimulation-induced corticomotor excitability and associated motor skill acquisition in chronic stroke. Stroke 37: 1471-1416.

5. Lefaucheur JP, Jarry G, Drouot X, Ménard-Lefaucheur I, Keravel Y, et al. (2010) Motor cortex rTMS reduces acute pain provoked by laser stimulation in patients with chronic neuropathic pain. Clin Neurophysiol 121: 895-901.

6. Bestmann S, Baudewig J, Siebner HR, Rothwell JC, Frahm J (2003) Subthreshold high-frequency TMS of human primary motor cortex modulates interconnected frontal motor areas as detected by interleaved fMRI-TMS. Neuroimage 20: 1685-1696.

7. Bestmann S, Baudewig J, Siebner HR, Rothwell JC, Frahm,J (2004) Functional MRI of the immediate impact of transcranial magnetic stimulation on cortical and subcortical motor circuits. Eur J Neurosci 19: 1950-1962.

8. Bohning DE, Shastri A, McConnell KA, Nahas Z, Lorberbaum JP, et al. (1999)A combined TMS/fMRI study of intensity-dependent TMS over motor cortex. Biol Psychiatry 45: 385-394.

9. Hanakawa T, Mima T, Matsumoto R, Abe M, Inouchi M, et al. (2009) Stimulusresponse profile during single-pulse transcranial magnetic stimulation to the primary motor cortex. Cereb Cortex 19: 2605-2615.

10. Rossini PM, Barker AT, Berardelli A, Caramia MD, Caruso G, et al. (1994) Noninvasive electrical and magnetic stimulation of the brain, spinal cord and roots Basic principles and procedures for routine clinical application. Report of an IFCN committee. Electroencephalogr Clin Neurophysiol 91: 79-92.

11. Denslow S, Lomarev M, George MS, Bohning DE (2005) Cortical and subcortical brain effects of transcranial magnetic stimulation (TMS)-induced movement: An interleaved TMS/Functional magnetic resonance imaging study. Biol Psychiatry 57: 752-760.

12. Eickhoff SB, Paus T, Caspers S, Grosbras MH, Evans AC, et al. (2007) Assignment of functional activations to probabilistic cytoarchitectonic areas revisited. Neuroimage 36: 511-521.

13. Logothetis NK, Pauls J, Augath M, Trinath T, Oeltermann A (2001) Neurophysiological investigation of the basis of the fMRI signal. Nature 412: 150-157.

14. Shmuel A, Yacoub E, Pfeuffer J, Van de Moortele PF, Adriany G, et al. (2002) Sustained negative BOLD, blood flow and oxygen consumption response and its coupling to the positive response in the human brain. Neuron 36: 1195-1210.

15. Smith AT, Williams AL, Singh KD (2004) Negative BOLD in the visual cortex evidence against blood stealing. Hum Brain Mapp 21: 213- 220.

16. Allison JD, Meador K, Loring DW, Figueroa RE, Wright JC (2000) Functional MRI cerebral activation and deactivation during finger movement. Neurology 54: $135-142$

17. Stefanovic B, Warnking JM, Pike GB (2004) Hemodynamic and metabolic responses to neuronal inhibition. Neuroimage 22: 771-778.

18. Kastrup A, Baudewig J, Schnaudigel S, Huonker R, Becker L, et al. (2008) Behavioral correlates of negative BOLD signal changes in the primary somatosensory cortex. Neuroimage 41: 1364-1371.

19. Pasley BN, Inglis BA, Freeman RD (2007) Analysis of oxygen metabolism implies a neural origin for the negative BOLD response in human visual cortex. Neuroimage 36: 269-276.

20. Shmuel A, Augath M, Oeltermann A, Logothetis NK (2006) Negative functional $\mathrm{MRI}$ response correlates with decreases in neuronal activity in monkey visual area V1. Nat Neurosci 9: 569-577.

21. Wassermann EM (1998) Risk and safety of repetitive transcranial magnetic stimulation: Report and suggested guidelines from the International Workshop on the Safety of Repetitive Transcranial Magnetic Stimulation, 1996 Electroencephalogr Clin Neurophysiol 108: 1-16.

22. Di Lazzaro V, Restuccia D, Oliviero A, Profice P, Ferrara L, et al. (1998) Magnetic transcranial stimulation at intensities below active motor threshold activates intracortical inhibitory circuits. Exp Brain Res 119: 265-268.

23. Fisher RJ, Nakamura Y, Bestmann S, Rothwell JC, Bostock H (2002) Two phases of intracortical inhibition revealed by transcranial magnetic threshold tracking. Exp Brain Res 143: 240-248.

24. Kujirai T, Caramia MD, Rothwell JC, Day BL, Thompson PD, et al. (1993) Corticocortical inhibition in human motor cortex. J Physiol 471: 501-519. 
Citation: Shimomura T, Fujiki M, Ohba H, Kochiyma T, Sugita K, et al. (2018) Contralateral Negative Bold Responses in the Motor Network during Subthreshold High-Frequency Interleaved TMS-fMRI over the Human Primary Motor Cortex. J Neurol Neurophysiol 9: 478. doi:10.4172/21559562.1000478

Page 8 of 8

25. Ziemann U, Lönnecker S, Steinhoff BJ, Paulus W (1996) Effects of antiepileptic drugs on motor cortex excitability in humans: a transcranial magnetic stimulation study. Ann Neurol 40: 367-378.

26. Fitzgerald PB, Fountain S, Hoy K, Maller J, Enticott P, et al. (2007)A comparative study of the effects of repetitive paired transcranial magnetic stimulation on motor cortical excitability. J Neurosci Method 165: 265-269.

27. Ferbert A, Priori A, Rothwell JC, Day BL, Colebatch JG, et al. (1992) Interhemispheric inhibition of the human motor cortex. J Physiol 453: 525546

28. Tergau F, Wanschura V, Canelo M, Wischer S, Wassermann EM, et al. (1999) Complete suppression of voluntary motor drive during the silent period after transcranial magnetic stimulation. Exp Brain Res 124: 447-454.

29. Brocke J, Schmidt S, Irlbacher K, Cichy RM, Brandt SA (2008) Transcranial cortex stimulation and fMRI: Electrophysiological correlates of dual-pulse BOLD signal modulation. Neuroimage 40: 631-643.

30. Chen R, Yung D, Li JY (2003) Organization of ipsilateral excitatory and inhibitory pathways in the human motor cortex. J Neurophysiol 89: 1256-1264.

31. Kukaswadia S, Wagle-Shukla A, Morgante F, Gunraj C, Chen R (2005) Interactions between long latency afferent inhibition and interhemispheric inhibitions in the human motor cortex. J Physiol 563: 915-924.

32. Ni Z, Gunraj C, Nelson AJ, Yeh IJ, Castillo G, et al. (2009) Two phases of interhemispheric inhibition between motor related cortical areas and the primary motor cortex in human. Cereb Cortex 19:1654-1665.

33. Palmer LM, Schulz JM, Murphy SC, Ledergerber D, Murayama M, et al. (2012) The cellular basis of $\mathrm{GABA}(\mathrm{B})$-mediated interhemispheric inhibition. Science 335: 989-993.

34. Mochizuki H, Furubayashi T, Hanajima R, Terao Y, Mizuno Y, et al. (2007) Hemoglobin concentration changes in the contralateral hemisphere during and after theta burst stimulation of the human sensorimotor cortices. Exp Brain Res 180: $667-675$

35. Lefaucheur JP (2006) Stroke recovery can be enhanced by using repetitive transcranial magnetic stimulation (rTMS). Neurophysiol Clin 36: 105-15.

36. Takeuchi N, Chuma T, Matsuo Y, Watanabe I, Ikoma K (2005) Repetitive transcranial magnetic stimulation of contralesional primary motor cortex improves hand function after stroke. Stroke 36: 2681-2686.

37. Kim YH, You SH, Ko MH, Park JW, Lee KH, et al. (2006) Repetitive transcrania magnetic stimulation-induced corticomotor excitability and associated motor skill acquisition in chronic stroke. Stroke 37: 1471-1476.

38. Chang WH, Kim YH, Bang OY, Kim ST, Park YH, et al. (2010) Long-term effects of rTMS on motor recovery in patients after subacute stroke. J Rehabil Med 42: $758-764$

39. Nambu A, Tokumo H, Takeda M (2002) Functional significance of the corticosubthalamo-pallidal 'hyperdirect' pathway. Neurosci Res 43: 111-117. 